\title{
What Lies at the Heart of Photobiomodulation: Light, Cytochrome C Oxidase, and Nitric Oxide-Review of the Evidence
}

\author{
Brendan J. Quirk, PhD, and Harry T. Whelan, MD
}

\begin{abstract}
Objective: The underlying mechanisms of photobiomodulation (PBM) remain elusive. The most attractive hypotheses revolve around the role of cytochrome c oxidase (CCO) and cellular energetics.

Background: No reliable demonstration of any PBM-related light-induced mechanistic effect on CCO has been reported. Studies on PBM have proven to be either nonreproducible, of questionable relevance, or involve wavelengths unlikely to be operative in vivo. The literature reveals very few demonstrable mechanistic light effects of any sort on CCO. Nitric oxide (NO) is involved in a number of the reported light effects on CCO. NO inhibits CCO at high reductive pressures by binding to the heme $\mathrm{a}_{3}$ moiety. This complex is white light labile. Methods: The reported photolability of the heme-NO complex seems to be a prime target for PBM studies, as removal of inhibiting NO from the active site of CCO could restore normal activity to inhibited CCO. Another aspect of CCO-NO chemistry has been revealed that shows intriguing possibilities.

Results: A novel nitrite reductase activity of solubilized mitochondria has been demonstrated attributable to CCO. NO production was optimal under hypoxic conditions. It was also found that $590 \mathrm{~nm}$ irradiation increased NO production by enhancing NO release. The presence of cellular NO has usually been considered metabolically detrimental, but current thinking has expanded the importance and the physiological roles of NO. Evidence shows that NO production is likely to play a role in cardioprotection and defenses against hypoxic damage.

Conclusions: Studies combining PBM and hypoxia also point to a connection between light irradiation, hypoxia protection, and NO production. This leads the authors to the possibility that the intrinsic nature of PBM involves the production of NO. The combination of $\mathrm{CCO}$ and hemoglobin/myoglobin NO production with photorelease of NO may constitute the heart of PBM.
\end{abstract}

Keywords: photobiomodulation, cytochrome c oxidase, nitric oxide

\section{Introduction}

A FTER MANY DECADES OF STUDY, the underlying mechanisms of photobiomodulation (PBM) remain elusive. Although the most attractive hypotheses revolve around the role of cytochrome c oxidase (CCO) and cellular energetics, no reliable demonstration of any PBM-related light-induced mechanistic effect on $\mathrm{CCO}$ has been reported. Indeed, this has been the bane of the entire field all along, as studies on PBM, most relating to epiphenomena and not mechanistically oriented, have proven to be either nonreproducible, of questionable relevance, or involve wavelengths unlikely to be operative in vivo.

\section{Review and Discussion}

As endogenous chromophores with relevant absorbances are relatively rare, $\mathrm{CCO}$, and other heme proteins, remains the best target for our investigations. A thorough reading of the literature though reveals very few demonstrable light effects of any sort on CCO. Gibson and Greenwood ${ }^{1,2}$ studied the white light flash photolysis of carbon monoxide (CO) from the active site of CCO. Bocian et al. ${ }^{3}$ found no photoreduction of oxidized CCO with $600 \mathrm{~nm}$ light. Babcock et al. ${ }^{4}$ noted that oxygen bound to the heme $a_{3}$ site of CCO was photolabile using light of $532 \mathrm{~nm}$. Hallen et al. ${ }^{5}$ showed light of $532 \mathrm{~nm}$ caused retrograde electron transfer

\footnotetext{
Department of Neurology, The Medical College of Wisconsin, Milwaukee, Wisconsin, USA.

(c) Brendan J. Quirk and Harry T. Whelan, 2020; Published by Mary Ann Liebert, Inc. This Open Access article is distributed under the terms of the Creative Commons Attribution Noncommercial License (http://creativecommons.org/licenses/by-nc/4.0/), which permits any non-commercial use, distribution, and reproduction in any medium, provided the original author(s) and the source are credited.
} 
from $\mathrm{CuB}$ in the absence of $\mathrm{CO}$ or oxygen in the $\mathrm{CCO} \mathrm{E}$ turnover state. Pastore et al. ${ }^{6}$ reported that $632 \mathrm{~nm}$ laser preirradiation causes improved cytochrome $\mathrm{c}$ oxidation and oxygen consumption in isolated CCO. Quirk and Whelan, ${ }^{7}$ however, showed no effect on the kinetics of cytochrome $\mathrm{c}$ oxidation by isolated CCO using 670 and $830 \mathrm{~nm}$ preirradiation, or $660 \mathrm{~nm}$ concurrent irradiation. Brooks et al. ${ }^{8}$ demonstrated autoreduction of the binuclear center with subsequent oxygen binding using light $<300 \mathrm{~nm}$.

Nitric oxide (NO) is involved in many of the reported light effects on $\mathrm{CCO}$. Boelens et al. ${ }^{9,10}$ reported, using cryogenic EPR, the photolability of heme $a_{3}{ }^{+2}-\mathrm{NO}$, using 595,560 , or $430 \mathrm{~nm}$ light, from $\mathrm{CCO}$ in the $\mathrm{R}$ state, and $\mathrm{CuB}^{+2}$-NO using 640 and $<400 \mathrm{~nm}$ light in the $\mathrm{O}$ and $\mathrm{E}$ turnover states. Sarti et al. ${ }^{11}$ also found that NO bound to the heme $\mathrm{a}_{3}$ site in the $\mathrm{R}$ state (reduced $\mathrm{CCO}$ ) is white light labile at high reductive pressures (high reduced cytochrome c levels) and room temperature.

Sarti also reports ${ }^{11}$ on the dual nature of NO inhibition of CCO. Brown ${ }^{12}$ showed that NO inhibits oxygen consumption of $\mathrm{CCO}$ in a reversible nature and in competition with oxygen. Again he showed ${ }^{13}$ NO rapidly and reversibly inhibited the steady state turnover of isolated $\mathrm{CCO}$, and the inhibition was competitive with oxygen. He found that $\mathrm{NO}$ binds with high affinity to the oxygen binding site of $\mathrm{CCO}$ when it is reduced. He hypothesized that NO functioned to regulate mitochondrial respiration and cell functions. Sarti found $^{11}$ that NO inhibits $\mathrm{CCO}$ at high reductive pressures by binding to the heme $a_{3}$. This complex is white light labile. At low reductive pressures, either high turnover or low reduced cytochrome c levels, NO inhibits by binding to oxidized $\mathrm{CCO}$ at the $\mathrm{CuB}$ center. The NO then becomes an electron donor to heme $\mathrm{a}_{3}$ or, ultimately, $\mathrm{CuA}$. In this manner, $\mathrm{NO}$ is oxidized to nitrite $\left(\mathrm{NO}_{2}{ }^{-}\right)$, which is not light labile. The nitrite formed eventually diffuses from the active site, allowing for more nitrite formation. Cooper ${ }^{14}$ further elaborated on the various reactions of NO with $\mathrm{CCO}$. The predominate reactions in vivo are the inhibition of $\mathrm{CCO}$ by the binding of $\mathrm{NO}$ to heme $\mathrm{a}_{3}$ or $\mathrm{CuB}$. He also reports on the formation of $\mathrm{NO}$ by $\mathrm{CCO}$ from nitrite, but does consider this unlikely to be important physiologically due to slow kinetics and poor binding of nitrite.

Therefore, the reported photolability of the heme $\mathrm{a}_{3}{ }^{+2}-\mathrm{NO}$ complex would seem to be a prime target for PBM studies, as removal of inhibiting $\mathrm{NO}$ from the active site of $\mathrm{CCO}$ could restore normal activity to inhibited CCO. Borutaite et al. ${ }^{15}$ found that the NO-induced inhibition of respiration in isolated mitochondria due to inhibition of cytochrome oxidase was acutely reversible by white light. Light alone had no effect on oxygen consumption, but did relieve the inhibition induced by the addition of dissolved NO gas. In our own laboratory, studies of oxygen consumption inhibition of isolated $\mathrm{CCO}$ with an NO donor show no effect of 670 or $830 \mathrm{~nm}$ irradiation (unpublished results).

Although the NO-inhibition relief hypothesis has by no means been exhaustively explored, another aspect of CCO-NO chemistry has been revealed that shows intriguing possibilities. The Poyton group ${ }^{16}$ reported a novel nitrite reductase activity of solubilized mitochondria attributed to $\mathrm{CCO}$. They found that NO is produced over a wide range of oxygen concentrations, with NO production increasing as oxygen levels and $\mathrm{pH}$ decrease. The NO production was optimal under hypoxic conditions. They also found that irradiation increased NO synthesis in an intensity-dependent manner, without affecting oxygen consumption. The best results were found with $590 \mathrm{~nm}$ light, and were operative at physiological nitrite levels. Poyton and Ball lay out the case $^{17}$ for considering PBM to be a consequence of this CCO-related NO synthase (NOS) activity. In their view, NO is implicated in PBM, NO is made from nitrite by $\mathrm{CCO}$ under both hypoxic and, to some degree, normoxic conditions, and light enhances this activity. They propose that $590 \mathrm{~nm}$ irradiation enhances the NO off rate, hence the nitrite on rate and, therefore, NO production.

The presence of cellular NO has usually been considered metabolically detrimental, but current thinking has expanded the importance and the physiological roles of NO. ${ }^{18,19}$ In particular, protection against ischemia/reperfusion damage seems to be mediated by the presence or production of NO. Kosaka ${ }^{20}$ concluded that NO suppresses oxidative agents and improves oxygen transport, and that NO bound to hemoglobin $(\mathrm{Hb})$ increases oxygen release. DeVisscher et al. $^{21}$ found that during reperfusion NO increased to what would be inhibitory levels in vitro, but found no inhibition of $\mathrm{CCO}$ in vivo after anoxia. Cooper ${ }^{22}$ maintains that NO inhibition of oxygen consumption can increase the zone of oxygenation from blood vessel walls and lower the number of hypoxic cells. Brown and Borutaite ${ }^{23}$ claim that $\mathrm{Hb}$ and myoglobin $(\mathrm{Mb})$ in the heart may supply $\mathrm{NO}$ at low oxygen levels. Umbreit ${ }^{24}$ shows that while oxyHb consumes NO, forming nitrite, deoxyHb can consume nitrite, producing NO. Burkard et al. ${ }^{25}$ found that overexpression of neuronal NOS in mitochondria is cardioprotective through inhibition of mitochondrial function and decrease of reactive oxygen species. Umbrello et al. ${ }^{26}$ present a theory of a role for NO in hypoxia involving energy supply-demand matching. During hypoxia, NO modulates energy metabolism, reducing oxygen consumption and promoting alternative pathways. Under hypoxic conditions, both $\mathrm{Hb}$ and $\mathrm{Mb}$ can reduce nitrite to NO. He presents four mechanisms for rebalancing oxygen supply and demand in hypoxia: (1) increased NO production by NOS, (2) increased reduction of nitrite to NO by hemes and pterins, (3) increased release of NO from storage pools, and (4) decreased destruction of NO by CCO. La Padula et al. ${ }^{27}$ found that rats exposed to simulated high altitude (hypobaric conditions) had increased NO production, leading to cardioprotection. It is noted also that native Tibetans have increased NO levels.

Thus evidence shows that NO production is likely to play a role in cardioprotection and defenses against hypoxic damage. Studies combining PBM and hypoxia also point to a connection between light irradiation, hypoxia protection, and NO production. Zhang et al. ${ }^{28}$ reported protection against hypoxia and reoxygenation injury in cardiomyocytes by $670 \mathrm{~nm}$ light in a manner that is dependent upon NO derived from NOS and non-NOS sources. The protective effects of irradiation were dependent on the presence of NO. Quirk et al. ${ }^{29}$ demonstrated the safety and efficacy of using $670 \mathrm{~mm}$ irradiation for cardioprotection in an in vivo rat ischemia model, and that this cardioprotection was dependent upon some factor present in blood, but not in perfusion buffer. Lohr et al. ${ }^{30}$ studied NO release from nitrosyl-Hb and $\mathrm{Mb}$, and found NO release to be four times faster with 
$670 \mathrm{~nm}$ irradiation. $\mathrm{Hb}$ nitrite reductase activity was found to be maximal at $\sim 50 \%$ oxygen saturation. This released NO may enhance the cardioprotective effects of nitrite. Keszler et al. ${ }^{31}$ found that diabetic mice, which failed other cardioprotective strategies, were protected by $660 \mathrm{~nm}$ irradiation, independent of NOS. Further, $660 \mathrm{~nm}$ light released $\mathrm{NO}$ from $\mathrm{HbNO}$ and $\mathrm{MbNO}$ in vitro. In 2018 using s-nitrosothiol and dinitrosyl iron compounds, she found $670 \mathrm{~nm}$ light the most effective in releasing NO, though only 670,740 , and $830 \mathrm{~nm}$ wavelengths were tested.

This "mini-review" of CCO-related light interactions, along with NO-CCO and other heme protein chemistry, leads the authors to the possibility that the intrinsic nature of PBM involves the production of NO. Indeed, the combination of $\mathrm{CCO}$ and $\mathrm{Hb} / \mathrm{Mb} \mathrm{NO}$ production with photorelease of NO may constitute the heart of PBM.

\section{Summary Points and Conclusions}

(1) Most published CCO light effects are not reproducible or not relevant (too short wavelength, for example).

(2) Internal electron transfers in $\mathrm{CCO}$ are not light affected.

(3) Cardioprotection appears to be non-NOS-mediated NO production related.

(4) NO is produced by (non-NOS) nitrite reduction to NO by $\mathrm{CCO}$ or $\mathrm{Mb} / \mathrm{Hb}$ in a low oxygen environment and (in the case of $\mathrm{CCO}$ ) high reductive pressure (high metabolic demand). Pathological conditions favor NO production.

(5) The $590 \mathrm{~nm}$ light disassociates NO from CCO (or $\mathrm{Mb} / \mathrm{Hb}$ ), enhancing $\mathrm{NO}$ production and effects. Increasing NO off rate increases NO production.

(6) Most epiphenomenon PBM studies seem to be problematic. Particularly so are the wide variety of wavelengths and power and dose applications reported. Some studies may be subject to wishful thinking, selective data analysis, small effect sizes, small sample sizes, or high variability. Also questionable, but not necessarily intrinsically false, are notions of delayed effects (preirradiation) and the idea of "best dose": that is, lower and higher doses are detrimental, not beneficial. Pulsing may also be questionable. None of these ideas have any solid mechanistic or theoretical basis.

(7) If the main light-induced effect is indeed enhanced NO off rate by $590 \mathrm{~nm}$ light, studies showing effects of $>590 \mathrm{~nm}$ light may be either faulty or the result of the lower tail end of larger bandwidth sources. Perhaps genuine PBM effects do not require high power, but sources centered off $590 \mathrm{~nm}$ need high power to achieve an adequate dose in the $590 \mathrm{~nm}$ area. Optimal wavelength, particularly for NO release, requires further extensive exploration. It is noted $^{32}$ that $590 \mathrm{~nm}$ is located at the edge of the so-called tissue transparency window.

(8) Hypoxic/pathological PBM studies may be more creditable.

(9) Therefore, we propose that the major effect of light (PBM) may be enhanced NO production by $\mathrm{CCO}$ or $\mathrm{Mb} / \mathrm{Hb}$ by reducing nitrite to NO. This enhancement is due to the increased disassociation of the produced
NO from the ferrous-nitrosyl active site. Increasing the off rate will shift the equilibrium more in favor of the product (NO). NO may then have various downstream prosurvival actions, such as metabolic regulation, increased oxygen availability, antiapoptotic effects, and other types of signaling.

\section{Author Disclosure Statement}

No competing financial interests exist.

\section{Funding Information}

This study was supported, in part, by The Bleser Endowed Chair of Neurology and The Chad Baumann Neurology Research Endowment to Harry T. Whelan, M.D.

\section{References}

1. Gibson QH, Greenwood C. Reactions of cytochrome oxidase with oxygen and carbon monoxide. Biochem J 1963; 86:541-554.

2. Gibson QH, Greenwood C. The spectra and some properties of cytochrome oxidase components. J Biol Chem 1964; 239:586-590.

3. Bocian DF, Lemley AT, Petersen NO, Brudvig GW, Chan SI. Resonance Raman spectra of cytochrome c oxidase. Excitation in the 600-nm region. Biochemistry 1979;18: 4396-4402.

4. Babcock GT, Jean JM, Johnston LN, Woodruff WH, Palmer G. Flow-flash, time-resolved resonance Raman spectroscopy of the oxidation of reduced and of mixed valence cytochrome oxidase by dioxygen. J Inorg Biochem 1985;23:243-251.

5. Hallen S, Oliveberg M, Brzezinski P. Light-induced structural changes in cytochrome c oxidase. Measurements of electrogenic events and absorbance changes. FEBSLett 1993;318:134-138.

6. Pastore D, Greco M, Passarella S. Specific helium-neon laser sensitivity of the purified cytochrome c oxidase. Int J Radiat Biol 2000;76:863-870.

7. Quirk BJ, Whelan HT. Effect of red-to-near infrared light on the reaction of isolated cytochrome c oxidase with cytochrome c. Photomed Laser Surg 2016;34:631-637.

8. Brooks JL, Sucheta A, Einarsdottir O. Light-induced spectral changes in fully oxidized cytochrome c oxidase in the presence of oxygen. Biochemistry 1997;36:6336-6342.

9. Boelens R, Rademaker H, Pel R, Wever R. EPR studies of the photodiassociation reactions of cytochrome $c$ oxidasenitric oxide complexes. Biochim Biophys Acta 1982;679: 84-94.

10. Boelens R, Wever R, Van Gelder BF, Rademaker H. An EPR study of the photodissociation reactions of oxidised cytochrome C oxidase-nitric oxide complexes. Biochim Biophys Acta 1983;724:176-183.

11. Sarti P, Giuffre A, Forte E, Mastronicola D, Barone MC, Brunori M. Nitric oxide and cytochrome c oxidase: mechanisms of inhibition and NO degradation. Biochem Biophys Res Commun 2000;274:183-187.

12. Brown GC, Cooper CE. Nanomolar concentrations of nitric oxide reversibly inhibit synaptosomal respiration by competing with oxygen at cytochrome oxidase. FEBS Lett 1994;356:295-298.

13. Brown GC. Hypothesis nitric oxide regulates mitochondrial respiration and cell functions by inhibiting cytochrome oxidase. FEBS Lett 1995;369:136-139. 
14. Cooper CE. Nitric oxide and cytochrome oxidase: substrate, inhibitor or effector? Trends Biochem Sci 2002;27: 33-39.

15. Borutaite V, Budriunaite A, Brown GC. Reversal of nitric oxide-, peroxynitrite- and S-nitrosothiol-induced inhibition of mitochondrial respiration or complex I activity by light and thiols. Biochim Biophys Acta 2000;1459:405-412.

16. Ball KA, Castello PR, Poyton RO. Low intensity light stimulates nitrite-dependent nitric oxide synthesis but not oxygen consumption by cytochrome c oxidase: implications for phototherapy. J Photochem Photobiol B 2011;102: 182-191.

17. Poyton RO, Ball KA. Therapeutic photobiomodulation: nitric oxide and a novel function of mitochondrial cytochrome C oxidase. Discov Med 2011;11:154-159.

18. Poyton RO, Castello PR, Ball KA, Woo DK, Pan N. Mitochondria and hypoxic signaling a new view. Ann NY Acad Sci 2009;1177:48-56.

19. Taylor CT, Moncada S. Nitric oxide, cytochrome c oxidase, and the cellular response to hypoxia. Arterioscler Thromb Vasc Biol 2010;30:643-647.

20. Kosaka H. Nitric oxide and hemoglobin interactions in the vasculature. Biochim Biophys Acta 1999;1411:370-377.

21. De Visscher G, Springett R, Delpy DT, Van Reempts J, Borgers M, van Rossem K. Nitric oxide does not inhibit cerebral cytochrome oxidase in vivo or in the reactive hyperemic phase after brief anoxia in the adult rat. J Cereb Blood Flow Metab 2002;22:515-519.

22. Cooper CE. Competitive, reversible, physiological? Inhibition of mitochondrial cytochrome oxidase by nitric oxide. IUBMB Life 2003;55:591-597.

23. Brown GC, Borutaite V. Nitric oxide and mitochondrial respiration in the heart. Cardiovas Res 2007;75:283-290.

24. Umbreit J. Methemoglobin-it's not just blue: a concise review. Am J Hematol 2007;82:134-144.

25. Burkard N, Williams T, Czolbe M, et al. Conditional overexpression of neuronal nitric oxide synthase is cardioprotective in ischemia/reperfusion. Circulation 2010;122: 1588-1603.

26. Umbrello M, Dyson A, Feelisch M, Singer M. The key role of nitric oxide in hypoxia: Hypoxic vasodilation and energy supply-demand matching. Antiox Redox Sig 2013;19: 1690-1710.

27. La Padula PH, Etchegoyen M, Czerniczyniec A, et al. Cardioprotection after acute exposure to simulated high altitude in rats. Role of nitric oxide. Nitric Oxide 2018;73: 52-59.

28. Zhang R, Mio Y, Pratt PR, et al. Near infrared light protects cardiomyocytes from hypoxia and reoxygenation injury by a nitric oxide dependent mechanism. J Mol Cell Cardiol 2009;46:4-14.

29. Quirk BJ, Sonowal P, Jazayeri M, Baker JE, Whelan HT. Cardioprotection from ischemia-reperfusion injury by nearinfrared light in rats. Photomed Laser Surg 2014;32:505-511.

30. Lohr NL, Keszler A, Pratt P, Bienengraber M, Warltier DC, Hogg N. Enhancement of nitric oxide release from nitrosyl hemoglobin and nitrosyl myoglobin by red/near infrared radiation: potential role in cardioprotection. J Molec Cell Cardiol 2009;47:256-263.

31. Keszler A, Brandal G, Baumgardt S, et al. Far red/near infrared light induced protection against cardiac ischemia and reperfusion injury remains intact under diabetic conditions and is independent of nitric oxide synthase. Front Physiol 2014;5:1-7.

32. Nioka S, Wen S, Zhang J, et al. Simulation study of breast tissue hemodynamics during pressure perturbation. Adv Exp Med Biol 2005;566:17-22.

Address correspondence to: Harry T. Whelan, MD

Bleser Professor \& Endowed Chair Department of Neurology

The Medical College of Wisconsin 8701 Watertown Plank Road Milwaukee, WI 83226

USA

E-mail: hwhelan@mcw.edu

Received: May 29, 2020.

Accepted after revision: June 8, 2020. Published online: July 20, 2020. 